\title{
Establishment of a minimally invasive cardiac surgery programme in Singapore
}

Theo Kofidis ${ }^{1}$, MD, fRCS, Guo Hao $\underline{\text { Chang }}^{1}$, MBBS, MRCSEd, Chuen Neng $\underline{\operatorname{Le}}^{1}$, MBBS, FAMS

\begin{abstract}
Although minimally invasive cardiac surgery is available in Singapore, it is not widely practised. Local surgeons have pioneered and introduced technologies in Singapore that were launched in the West to address various heart pathologies. However, in spite of the possibilities, the unique position and function of Singapore in the region, the structure and dynamics of cardiothoracic surgery practice in government centres and private practice, and the overall small volume of patients in the country may pose limitations in the thriving and sharing of such new technologies. Herein, we give an account of our progress and accomplishments with regard to minimally invasive cardiac surgery in Singapore and indicate areas of improvement that have further potential for synergy and growth.
\end{abstract}

Keywords: aortic valve surgery, coronary artery bypass graft surgery, minimally invasive cardiac surgery, mitral valve surgery

\section{INTRODUCTION}

As a prologue to our discussion about minimally invasive cardiac surgery, we may resolve the nomenclature of the field by questioning the accuracy of the term 'minimally invasive'. In etymological terms, it denotes that which is most minimal on a comparative scale. With advancing technology and surgical skill, cardiac surgery procedures are becoming less invasive. Recently, Gerosa of the University of Padova, Italy, introduced the term 'microsurgery', referring to transapical repair of the mitral valve on the beating heart using Neochord implantation. ${ }^{(1}$ To spin the 'neologism' or 'neo-terminology' game further, one could assume that the least invasive of all cardiac procedures, which are and remain catheter-based procedures, should be called 'nanoinvasive'. Hence, the term 'minimal' could not withstand criticism, and we may wish to adopt the overarching term 'less invasive' - as opposed to 'classical cardiac surgery', which describes the field, median sternotomy and the use of cardiopulmonary bypass.

Less invasive cardiac surgery describes a group of alternative incisions or techniques that aim to reduce the surgical trauma, potential complications and burden of slow recovery following heart surgery. It is widely utilised in advanced centres in the West, China, Korea and Japan, ${ }^{(2-6)}$ and facilitates and encompasses procedures on heart valves, coronary arteries, arrhythmias and heart failure in various permutations. The most sublime and advanced forms of less invasive cardiac surgery can be said to be hybrid procedures, which are executed by heart surgeons and cardiologists together or in sequence. However, there are still arguments in favour of the old, familiar procedure, median sternotomy, which provides safe and reproducible access. Another limitation to the introduction and safe, widespread usage of such new technologies and gadgets is the relatively small number of cardiac surgery patients per surgeon in Singapore, particularly in the private sector. A further impediment is the natural course of action and resistance, otherwise known as entropy, which is habitual and a well-known impediment to progress and evolution. A similar paradigm of progress occurred in laparoscopic and video-assisted thoracoscopic surgeries: the first laparoscopic surgery was done in 1987, but only in 1991 did most general surgeons accept the procedure as the future direction for gallbladder surgery ${ }^{(7)}$ Less invasive cardiac surgery has, in fact, fulfilled the primary Hippocratic principle of primum non nocere (first, do no harm). Box 1 shows a non-exhaustive list of its advantages.

We herein give an account of our progress and accomplishments with regard to less invasive cardiac surgery in Singapore and indicate areas for consideration when establishing a similar programme. This article reflects our current knowledge of less invasive cardiac surgery, although some accomplishments elsewhere may have escaped our attention. We have been actively pursuing innovation and progress in an overall shrinking field, and consider it of paramount importance that the collegial audience is cognisant of and offers their patients all possible individualised options.

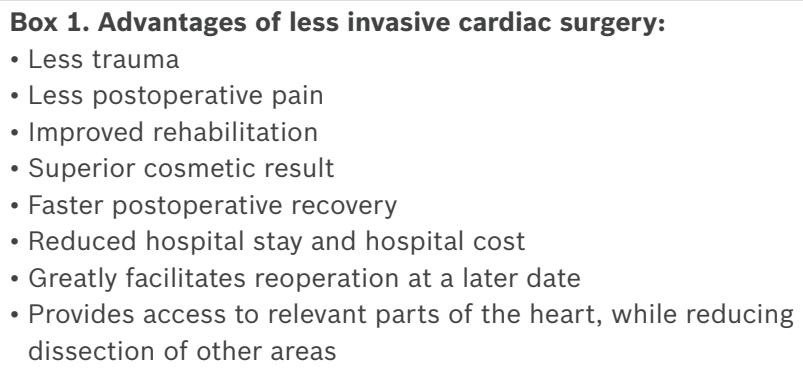

\section{OUR EXPERIENCE}

We have contributed to the introduction or wider establishment of a number of less invasive procedures or technologies (Fig. 1) - the first sutureless heart valve implantation, first same-stop hybrid procedure for coronary revascularisation, first full coronary revascularisation through a small anterolateral thoracotomy, first 

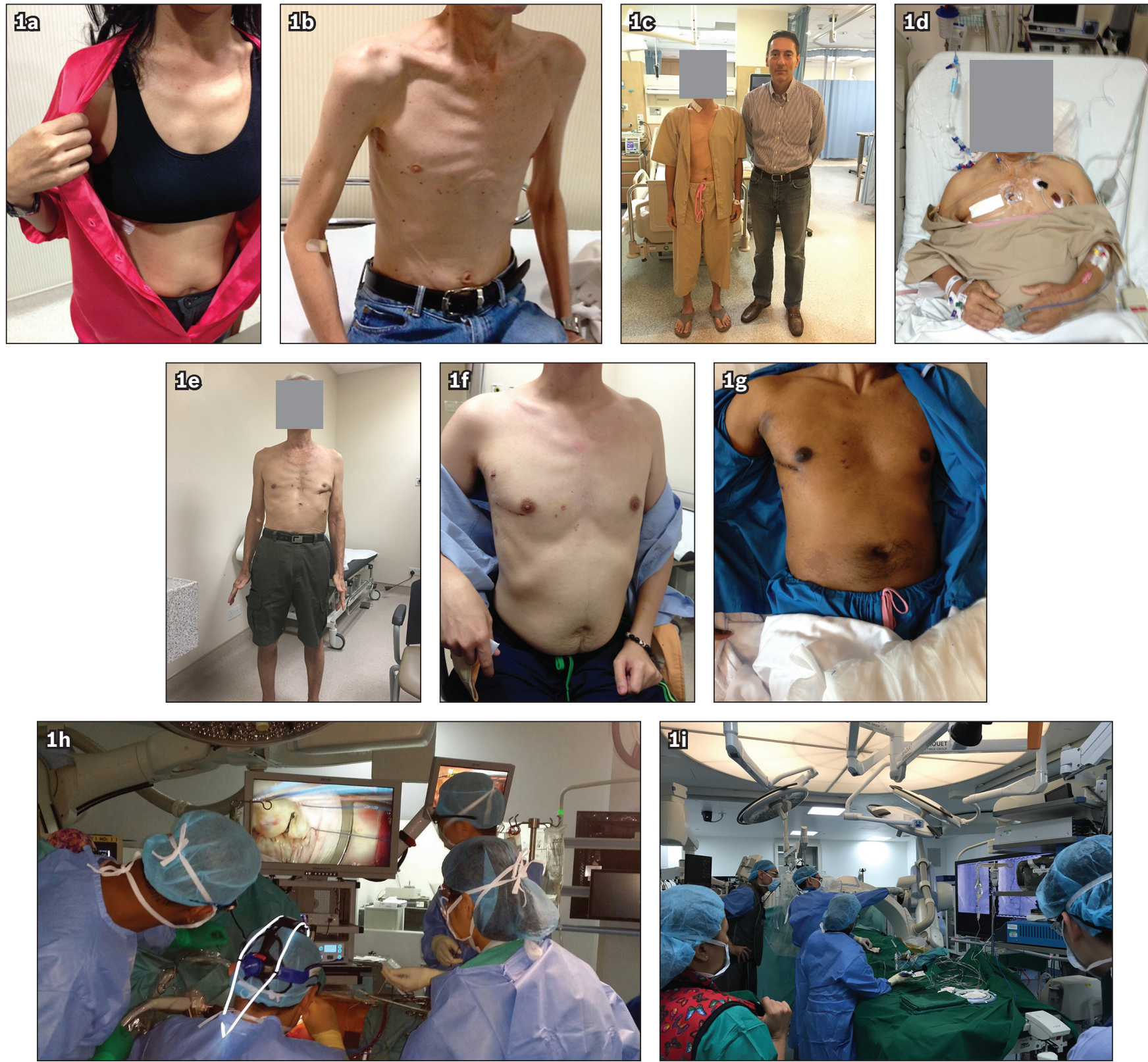

Fig. 1 Photographs show novel procedures or technologies that were introduced or adopted by our team and offered on a regular basis: (a) heart tumour removal through minimally invasive cardiac surgery; (b) single-incision minimally invasive cardiac surgery (SIMICS) developed at the National University Heart Centre; (c) first one-stop hybrid coronary artery bypass grafting (CABG) procedure in Southeast Asia; (d) first mini-aortic valve replacement (mini-thoracotomy) in Southeast Asia; (e) first McGinn full CABG in Asia; (f) mini-mitral valve surgery; (g) mini-tricuspid valve surgery without heart arrest; (h) first three-dimensional minimally invasive heart surgery in Asia, done via SIMICS; and (i) hybrid CABG procedure.

multiple heart valve and/or atrial septal defect closure procedure through the same mini-thoracotomy, first right-anterior minithoracotomy for an aortic valve replacement, first combined open-heart surgery and bypass surgery through the same work port from the right side, first utilisation of three-dimensional visualisation system, first automated knotting and tying device in cardiac surgery, and single-incision minimally invasive cardiac surgery (SIMICS; Fig. 1h). SIMICS is a less invasive cardiac surgery procedure that has rendered the robotic approach obsolete in our practice. It eliminates ports and a femoral incision for cannulation, and remains fast and less expensive when compared to robotic procedures.

Our efforts to provide this service in Singapore in a reproducible form were not easy, requiring more than eight years, a committed team of anaesthetists, nurses, perfusionists and surgeons, constant interaction and alliance with the world's best centres in the field, and constant battle with entropy. Only now is the programme being acknowledged as a strong platform for heart treatment in the country and region, attracting trainee surgeons from a number of countries and functioning as a training and proctorship hub.

We also established a plethoric cardiovascular animal laboratory (that eventually became one of the most advanced in the country) embedded within our campus in the immediate proximity of the National University Hospital, Singapore. This laboratory was funded by sequential grants provided by the National Medical Research Council and National Research Foundation, which have generously supported our efforts over 
Box 2. Some dos and don'ts for less invasive cardiac surgery:

Dos

- Bear in mind: less invasive cardiac surgery is heart surgery

first - the same principles apply.

- Train your team.

- Individualise approach and access.

- Take good informed consent.

- Avoid extreme anatomy and very flat-chested, tiny patients.

- Consider cost.

Don'ts

- Don't be fanatic about it.

- Don't compromise the quality of repair for the draw of less invasive cardiac surgery.

- Don't turn minimal into maximal.

the last ten years. In it, a team of 22 clinicians, scientists and students have worked under the first author's supervision to produce new tools, try out new techniques and reduce the burden for the patient, by experimenting with small and large animals. All these components are key ingredients of this programme. Other components include clinical applications, local and global alliances, industry support, teaching and proctorships, and research.

\section{LESSONS LEARNT}

It is useful to understand the key ingredients of a successful less invasive cardiac surgery programme, which is commonly associated with a short learning curve. An inexperienced cardiac surgeon who cannot master complications and is not trained to perform complex procedures is unlikely to be able to transit effectively to the less invasive platform. On the other hand, a good cardiac surgeon usually transits into less invasive cardiac surgery much more easily than he originally thinks and very soon overcomes the fear of the unknown. With advanced technology, procedures that used to be impossible can now be safely performed. Indeed, contraindications from five years ago are no longer so. Box 2 summarises some of the dos and don'ts for less invasive cardiac surgery.

The belief that the establishment of a thriving less invasive programme is financially unmanageable is unfounded, particularly in a country such as Singapore. The first author has helped start such programmes with very little investment and tools throughout his travels, proctorship and preceptorship. In Singapore, it costs much less to purchase the full set of equipment, including the entire platform, for less invasive cardiac surgery when compared to the costs involved for the robotic approach. The latter may aggravate the overall cost into a few million dollars, but this additional cost is not necessary, as manual less invasive procedures are faster, easier, didactically more comprehensive and reproducible. As the authors have demonstrated in practice, they can also be less invasive than the robotic procedure. Preliminary findings from our unpublished study suggest that despite the utilisation of new gadgets and tools, less invasive heart surgery resulted in hospital stays per patient that were approximately two days shorter and fewer corresponding patient bills, for the majority of procedures.

\section{WHY I DO LESS INVASIVE HEART SURGERY}

This excellent question, which was posed to the author as the title for a workshop in the domain of computed tomography surgery, deserves an honest response. On a lighthearted note, the intonation of the sentence is key: 'Why I do less invasive heart surgery' versus 'Why I do less invasive heart surgery'. The driving forces for this type of surgery, and not 'Why on earth are we troubling ourselves with the headache of innovation?', should be as follows:

- It is here to stay and is the present and future.

- It is developing into the new gold standard.

- $\quad$ Technology is changing and patients are changing, too. They expect less invasive cardiac surgical techniques with expeditious recovery.

- It is a good challenge for growth and self-improvement.

- Medicine is in constant flux, striving to help without harming.

- It provides fewer complications and faster recovery in most cases.

- It allows customisation of patients' treatment, which is no longer 'one size fits all'.

- It is a good platform for synergy with the cardiologist within the multidisciplinary team.

- It gives Singapore and my institution the edge by offering an option to patients that entails greater benefits.

- It is what I would hope to receive for myself (or my family) in a similar situation.

- Our duty, as surgeons and academics, is to seek better solutions and stand for controversy and discussion, as well as teach and facilitate for others to follow.

- I want to see the individual surgeon and the collegium of heart surgeons synergise toward greater heights, with a newfound sense of accomplishment and ambition.

Similarly, we can answer the question 'Why not do less invasive cardiac surgery?' as follows:

- It is not expensive.

- It is not as traumatic as classical surgery.

- It is not difficult.

- It is not experimental.

\section{CONCLUSION}

Less invasive heart surgery is a great opportunity and not a threat for our profession, and can help provide an edge for our hightech healthcare system without colliding with the principles of value-driven outcomes. It also facilitates better collaboration and closer synergy with our gatekeepers, the cardiologists. Less invasive heart surgery is a plethoric platform that opens new pathways for cooperation within the multidisciplinary team, offering customisable care and attracting more job opportunities as well as patient volumes for a type of surgery that is right for the patient, if the patient qualifies for it. We recognise the tremendous potential of less invasive heart surgery in Singapore to elicit more synergy, fusion, and exchange of knowledge and skills; provide 
space for noble competition; and help the field thrive again in the regional context, with Singapore re-assuming a lead role despite lower case volumes. To conclude, we can perhaps reflect upon a quote from ancient Greek philosopher Aristotle: "The good (i.e. greatness/excellence) does not lie in the 'Much', but in the Who and How'".

\section{REFERENCES}

1. Colli A, Manzan E, Zucchetta F, et al. Transapical off-pump mitral valve repair with Neochord implantation: early clinical results. Int J Cardiol 2016; 204:23-8.

2. Bang JH, Kim JW, Lee JW, et al. Minimally invasive approaches versus conventional sternotomy for aortic valve replacement: a propensity score matching study. Korean J Thorac Cardiovasc Surg 2012; 45:80-4.

3. Park WK, Lee JW, Kim JB, et al. Outcomes of surgical atrial fibrillation ablation: the port access approach vs. median sternotomy. Korean J Thorac Cardiovasc Surg 2012; 45:11-8

4. Katayama I, Tanaka M, Noguchi K, et al. [Early results of minimally invasive cardiac surgery (MICS) mitral valve surgery with 51 cases]. Kyobu Geka 2015; 68:16-22. Japanese.

5. Gong W, Cai J, Wang Z, et al. Robot-assisted coronary argery bypass grafting improves short-term outcomes compared with minimally invasive direct coronary artery bypass grafting. J Thorac Dis 2016; 8:459-68.

6. Zhang L, Cui Z, Song Z, et al. Minimally invasive direct coronary artery bypass for left anterior descending artery revascularisation - analysis of 300 cases. Wideochir Inne Tech Maloinwazyjne 2016; 10:548-54.

7. Osborne DA, Alexander G, Boe B, Zervos EE. Laparoscopic cholecystectomy: past, present, and future. Surg Technol Int 2006; 15:81-5. 